\title{
Simulative Investigation of Flat Fading Wireless Channels in terms of First Order and Second Order Statistics
}

\author{
Joy Karan Singh \\ ECE Department \\ Guru Nanak Dev University, Regional Campus \\ Jalandhar, India
}

\author{
Jyoteesh Malhotra, Ph.D \\ ECE Department \\ Guru Nanak Dev University,Regional Campus \\ Jalandhar, India
}

\begin{abstract}
Second order statistics provides an efficient representation of a fading channel and plays a major role in the designing and evaluation of the wireless communication systems. This paper presents a channel model framework for the implementation of important second order statistical parameters, in particular the Level Crossing Rate (LCR) and the Average Fade Duration (AFD). In addition to this, other key first order statistics including Doppler Shift, CDF PDF, BER are also analysed. It also analyses the variations of LCR and AFD in multiple scattering environment .The analysis references to the well known Clarkes Model which has been utilised to plot the variations in key parameters.
\end{abstract}

\section{Keywords}

Doppler Effect, AFD, LCR, BER, Fading Channel model, Diversity Systems

\section{INTRODUCTION}

Channel Model can effectively demonstrate the channel behaviour based on its fading statistics. The purpose of modelling is to compute and estimate the various first and higher order fading channel statistical parameters. These channel parameters are derived from the model for performance evaluation and design of wireless communication system. Some of these parameters are Level Crossing Rate (LCR), outage time, and outage probability, distribution of LCR and sojourn time. Outage time is the time duration for which channel suffers with outage. Outage probability shows the probability of outage occurrence. Other interesting parameter is LCR which gives the number of fades below a certain level during fixed time interval. The probability distribution of LCR further describes the fading rate of the channel. The contribution of analytical approximation using level crossing rate is important for real time application. The duration of a signal fade determines the probable number of signalling bits that will be lost during the fade. This can be used to calculate BER. Sojourn time is described in terms of number of time steps for which channel remains in same state. Its distribution demonstrates the rate of change of fading. Channel model can be further used for estimating the probability of rare events like the deep fades, the probability of channel recovery from deep fading and the critical outage time over which channel can survive.

In the case of mobile communications, fading is a common phenomenon. Second order statistics are used in the design evaluation of mobile systems. Diversity methods are used to remove fading. Diversity concept uses multiple antennas and adaptive receivers. In context to this, the constituents of the transmitted signals at the receiver end have different fading distributions which are mutually correlated.. The LCR is used for finding the fading rate and is an important parameter. The LCR is a second order statistic of this fading and is influenced by the speed of the mobile receiver. Other associated parameters of interest encompass the Auto Correlation and the Cross Correlation.

Various scenarios can be considered for getting acquainted with multipath propagation. The Doppler Effect (or Doppler Shift) is a phenomenon in which frequency of a wave changes for an observer moving relative to the source of the wave. The random processes associated with fading channels are usually characterized by their probability density function or cumulative distribution function. The second-order statistics of fading channels signifies information associated with not only with the scattering environment, but also with the the system dynamics. The LCR provides information about how often the envelope fading crosses a certain threshold. The Average Fade Duration provides information related with the amount of time that the envelope remains below this threshold. This paper simulates the impact of number of scatterers on the Doppler Spectrum and the associated second order statistics including LCR and AFD for the low and high density scattering environments.

As the lower order statistics are sometimes insufficient in analysing the signals in detail, there is a need to review the higher order statistics like the LCR and AFD as they provide a better analytical assessment for the fading channels. Additionally, the Gaussian-distributed signals provide an interesting characteristic of disappearing at higher orders. As the noise and interference environment is Gaussiandistributed, the higher-order statistics offers an additional method of noise reduction \& interference mitigation and can be used to generate a filtering algorithm. LCR and AFD have found many applications in the modelling and design of wireless communication systems. LCR can be defined as the number of times per unit duration that the envelope of a fading channel crosses a given value in the negative direction. AFD corresponds to the average length of time the envelope remains under the threshold value once it crosses it in the negative direction.

\subsection{Background}

\section{a) First order statistics}

The probability density function (pdf), of the Rayleigh distribution is given by -

$$
f(r)=\frac{r}{\sigma^{2}} \exp \left(-\frac{r^{2}}{2 \sigma^{2}}\right) \quad \text { for } r \geq 0
$$


By integrating the pdf, the cumulative distribution function (CDF), can be obtained, i.e.,

$$
\operatorname{CDF}(R)=\operatorname{Prob}(r \leq R)=\int_{0}^{R} f(r) d r=1-\exp \left(-\frac{R^{2}}{2 \sigma^{2}}\right)
$$

\section{Bit error rate (BER)}

Bit error rate is a useful parameter that is used for accessing the performance when data is transmitted. BER is applicable to radio data links, IP networks and fibre optic data systems. Errors can be introduced when data is transmitted from one system to other. BER is used to access the end to end performance of telecommunication networks comprising of the transmitter, receiver and the intermediate medium.

\section{b). Second order statistics}

The received signal envelope experiences fading whenever the transmitter or the receiver moves. In addition, the surroundings of the communication channel also change with time that can also account for fading. The number of times the envelope crosses a threshold level in a unit of time signifies the signal fade rate. The level crossing rate is directly related to the speed of the receiver as a faster receiver will modify the conditions of the channel more rapidly. Several efforts to model the crossing rate statistically have been tried, but due to absence of the second order statistics, an empirical modelling is anticipated. The level crossing rate at envelope $\mathrm{R}$ is defined as the rate at which a fading signal envelope crosses level $\mathrm{R}$ in any direction. In general this has been represented as:

$$
\int_{0}^{\infty} r p(R, r) d r
$$

With the $p(R, r)$ representing the joint probability density function of $r$. The average duration of fades is the average length of time for which the signal envelope remains less than threshold of signal level. ADF may be used to analyse the impact of fading on digital transmission systems operating in fading mobile radio systems. In these systems, ADF may serve as a guide to the design of packet radio systems.

\subsection{Related work}

Victor M.hinostroza [1] proposed that each environment presents different behaviour about LCR. Even in similar spaces such as floor penetration and inside a building, the behaviour is quite different. With regards to the ADF, it shows that similar environments ie floor penetration and inside building have similar ADF behaviour.

Ali Abdi [2] proposed and expressed LCR in terms of joint characteristic function. This new formula is useful in many cases where the joint $\mathrm{cf}$ is is simpler to derive than the associated joint $\mathrm{cf}$.

Cheng -Xiang Wang[3] proposed a simulation model for nakagami -hoyt fading channel model. Various formulas for AFD LCR PDF were derived.

Gulzaib[4] studied lcr and adf and other first order statistics for $\mathrm{N}$-nakagami-m channels.

Aleksandra M. Mitic[5] proposed that for shadowing and fading in mobile communication, statistical characterization is important and in this paper that is computed for Ricean fading channels.
Nsiba mandel[6] proposed that with increase in Doppler frequency value of LCR increases while AFD decreases.

Zoran hadzi velkov[7] proposed a framework for analysis of second order statistics such as lcr afd for amplify and forward multi-hop Rayleigh fading channels. Various examples were taken and computer and numerical simulation verify the accuracy of the proposed mathematical analysis and the tightness of the approach is result of this.

Khedhiri,R [8] studied the first order and second order statistics for selection combing for nagakami-m fading channels

Yilmaz,F[9] analyzed millimetre wave fading channels and free space optical channels.Probability density function, cumulative distribution function, level crossing rate and average fade duration, moments, amount of fading and average capacity are derived

Anjana jain[10] analyzed fading statistics of Nakagami channel,various statistics such as tolerance time are analyzed.

Chau yawgeng[11] analyzed Rayleigh fading channels where different relevant second-order cross-correlation functions of in-phase and quadrature components of the cascaded Rayleigh channels are derived.

Milos bandjur[12] in this paper second order statistics of dual branch selection combining (SC) diversity receiver operating over $\mathrm{k}-\mu$ multipath fading environment is considered.

Tasos[13] proposed expressions for LCR and AFD for. $\alpha-\kappa-$ $\mu$ fading channel.

Section 1 of this paper deals with the basic channel fading model system model used. Section 2 deals with the simulation setup and values of parameters used. Section 3 deals with results and discussions of the setup implemented. Section 4 summarises the study with conclusion and references.

\section{CHANNEL MODEL}

The use of fading channel models is to determine the effects of second order and first order statistics in transmission of information over the air interface in broadcasting as well as cellular communication. Figure below illustrates the basic block diagram of proposed multipath fading channel model.

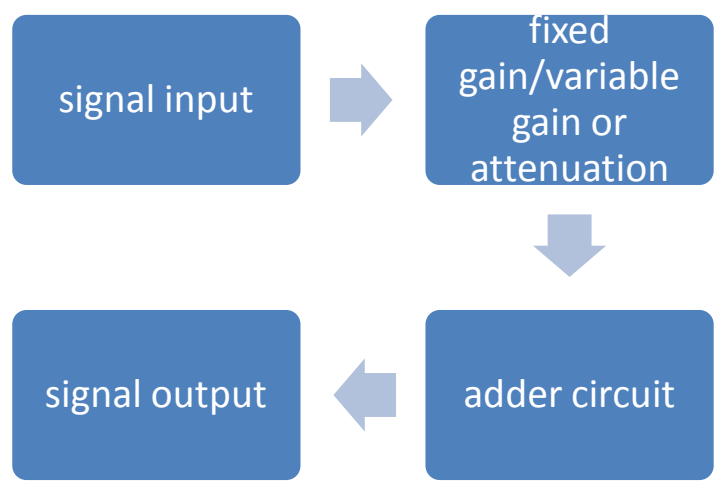

\section{Figure 1 - Fading Channel Model}

The input signal is passed through two different gains: First part covers the fixed gain, while the second represents a variable gain. This model assumes that the magnitude of a signal that has passed through a communication channel (also 
called a transmission medium) will fade according to the Rayleigh distribution or will have random variations.

$$
\begin{aligned}
\mathrm{y}(\mathrm{t})=\mathrm{g} 1(\mathrm{t}) * \mathrm{~s}(\mathrm{t}) & +0.5[\tau * \mathrm{~g} 2 * \mathrm{~s}(\mathrm{t})]+0.25[\tau * \mathrm{~g} 2 * \mathrm{~s}(\mathrm{t})] \\
+ & \mathrm{n}(\mathrm{t})
\end{aligned}
$$

Where, $y(t)$ is output signal, $s(t)$ is input signal, $\tau$ is delay or phase shift, g1 is fixed gain, g2 is variable gain and $\mathrm{n}(\mathrm{t})$ represents noise. It is a statistical model used to measure the effect of a propagation environment on a radio signal, such as that used in case of wireless devices. We have used the following function to generate noise.

$$
\text { syntax: } y=A W G N(x, S N R, \text { measured })
$$

\section{SIMULATION ENVIROMENT AND METHODOLOGY}

In this paper, we will have endeavoured to reproduce the Clarke Model using the multiple point scatterer approach. Normalization of signals has been performed with respect to free space. To simulate the low density and high density environments, we have set them on a circle about MS which are $1 \mathrm{~km}$ from origin. Two scenarios have been considered in this case with the number of scatterers being set to 100 and 5000 respectively.

Subsequent to this, other parameters frequently found in the literature are presented with regards to the simulation results. The two key parameters focused are the level crossing rate (lcr) and the average fade duration (afd). These parameters are often called second-order statistics as they depend on the mobile speed. Simulation parameters for the two scenarios have been illustrated in the table below

Table 1 - Parameters for Simulation

\begin{tabular}{|l|l|l|l|}
\hline \multicolumn{1}{|c|}{ Scenario1 } & & \multicolumn{1}{|c|}{ Scenario2 } & \\
\hline Reference Model & Clarkes & Reference Model & Clarkes \\
\hline $\begin{array}{l}\text { Bs distance from } \\
\text { origin }\end{array}$ & $1 \mathrm{~km}$ & $\begin{array}{l}\text { Bs distance from } \\
\text { origin }\end{array}$ & $1 \mathrm{~km}$ \\
\hline No of scatterers & 100 & No of scatterers & 5000 \\
\hline No of FFT points & 1024 & No of FFT points & 1024 \\
\hline No of samples & 1000 & No of samples & 5000 \\
\hline Carrier frequency & 2000 & Carrier frequency & 2000 \\
\hline MS1 speed & 10 & MS1 speed & 10 \\
\hline
\end{tabular}

Algorithm for Doppler spectrum

Step 1 - Creation of Doppler objects

Step 2 - Duplication of Doppler objects

Step 3 - View and change of Doppler properties

Step 4 - Use of Doppler objects within channel objects

Step 5 - Configuring of channel objects

\section{Algorithm for cdf and pdf}

Step 1 - Calculation of pdf

Step 2 - Plot histogram representing cdf and pdf

Step 3 - Verification of histogram representing Rayleigh distribution.

Step 4 - Check for compliance for the fit

Step 5 - Representation against theoretical and scenario plots

\section{BER Script Methodology}

Step 1 - Generation of binary sequence with +1 s and -1 s

Step 2 - Multiplication of channel with the symbols followed by addition of white Gaussian noise.

Step 3 - Equalization of (divide) the received symbols with the known channel at the receiver

Step 4 - Execution of hard decision decoding and counting of the bit errors

Step 5 - Repetition for multiple values of $\frac{E_{b}}{N_{0}}$ and plotting the scenario simulation along with the theoretical results.

\section{RESULTS AND DISCUSSIONS}

This paper focuses on two scenarios to analyse the impacts of density variation with regards to the key second order statistics pertaining to flat fading.

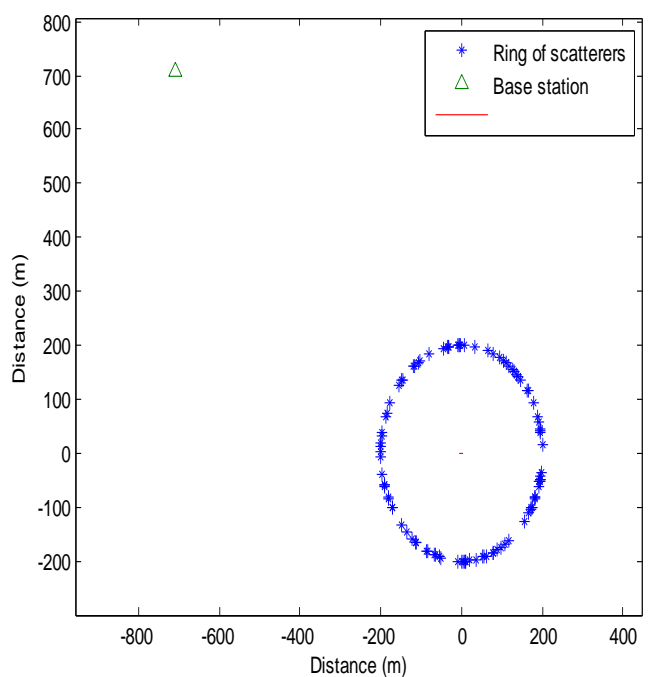

Figure 4a - Scenario with low density carrier

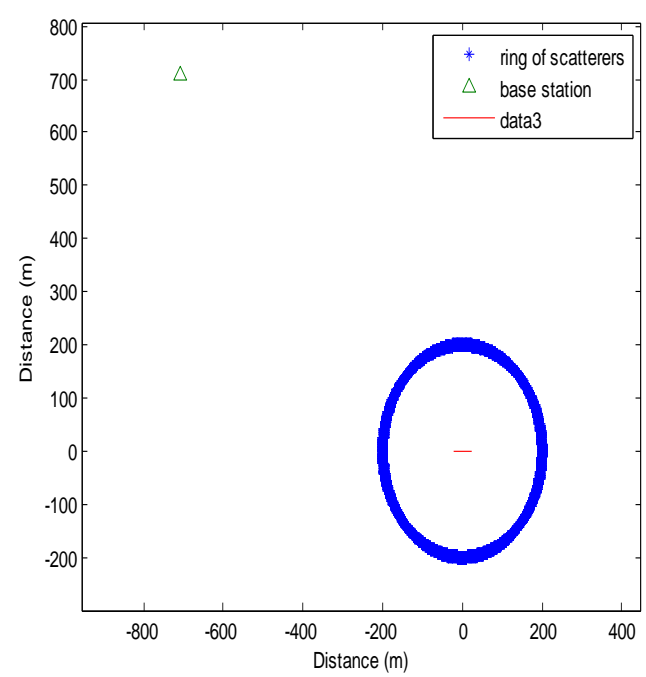

Figure 4b - Scenario with high density carriers 


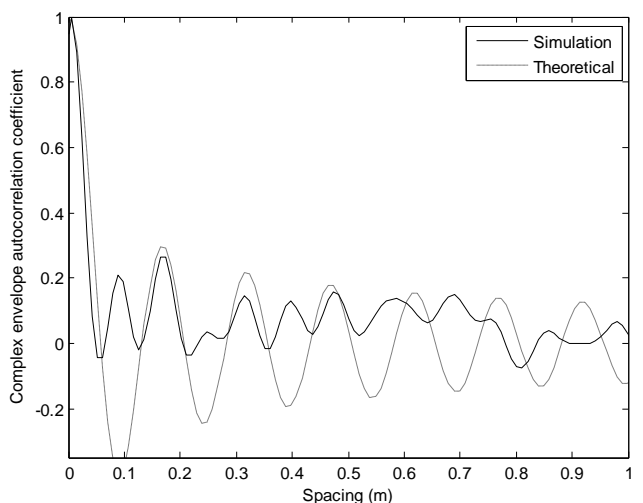

Figure 4c - Autocorrelation Coefficient
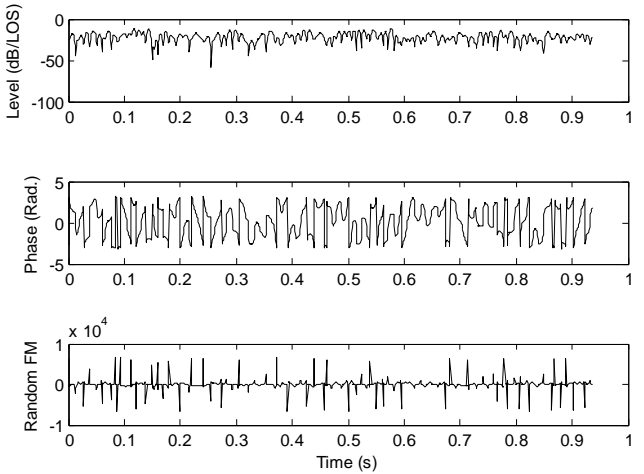

Figure 4d - Signal Level, Phase Variations, Random FM

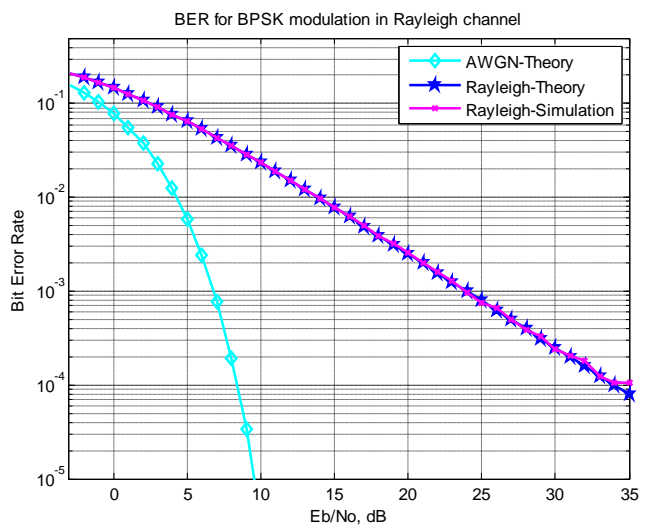

Figure 4e - BER for BPSK Modulation

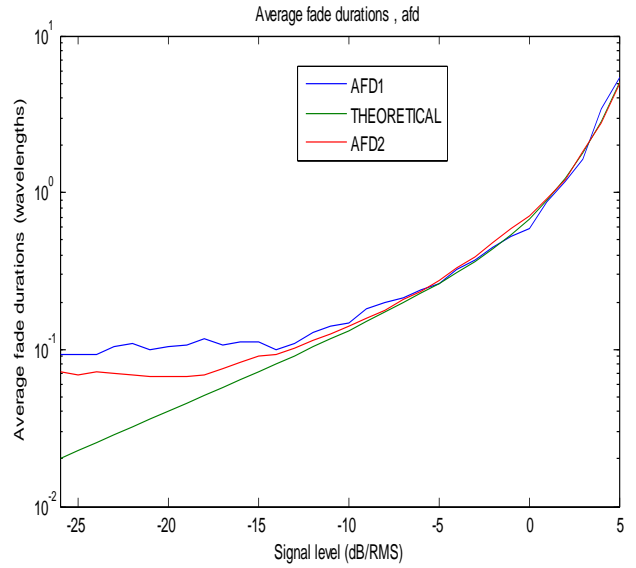

Figure 4f - AFD Variations

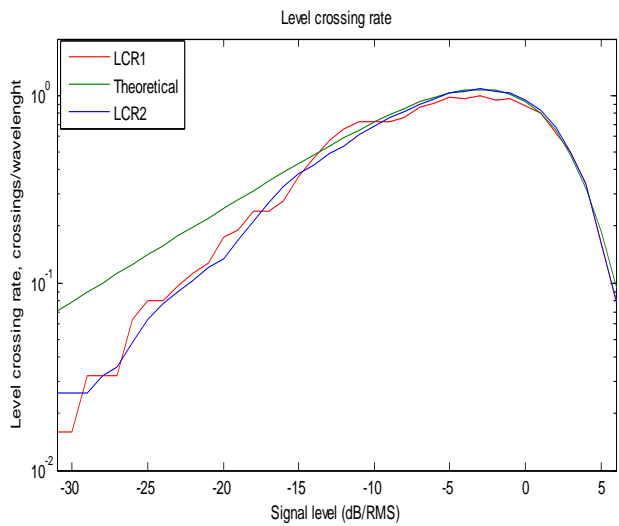

Figure 4g - LCR variations

Table 2- AFD Calculations for 2 Scenarios / Plot 4f

\begin{tabular}{|c|c|c|c|}
\hline $\begin{array}{c}\text { AFD1 } \\
\text { (Low Density) }\end{array}$ & $\begin{array}{c}\text { AFD2 } \\
\text { (High density) }\end{array}$ & Variation & \% Variation \\
\hline 0.0014 & 0.0019 & 0.0005 & 35.17 \\
\hline 0.0014 & 0.0019 & 0.0005 & 35.17 \\
\hline 0.0012 & 0.0014 & 0.0002 & 16.66 \\
\hline 0.0012 & 0.0014 & 0.0002 & 16.66 \\
\hline 0.0012 & 0.0012 & 0.0000 & 0 \\
\hline 0.0012 & 0.0012 & 0.0000 & 0 \\
\hline 0.0011 & 0.0011 & 0.0000 & 0 \\
\hline
\end{tabular}

Inference for AFD Variance - In high density scattering environment the value of AFD increases with increase in signal level.

Table3 - LCR Calculations for 2 Scenarios / Plot 4g

\begin{tabular}{|c|c|c|c|}
\hline $\begin{array}{c}\text { LCR1 } \\
\text { (Low Density) }\end{array}$ & $\begin{array}{c}\text { LCR2 } \\
\text { (High density) }\end{array}$ & Variation & \% Variation \\
\hline 0.0160 & 0.0032 & 0.0128 & 80 \\
\hline 0.0160 & 0.0032 & 0.0128 & 80 \\
\hline 0.0160 & 0.0032 & 0.0128 & 80 \\
\hline 0.0160 & 0.0032 & 0.0128 & 80 \\
\hline 0.0160 & 0.0032 & 0.0128 & 80 \\
\hline
\end{tabular}

Inference for LCR Variance - In high density scattering environment the value of LCR decreases with increase in signal level.

\section{a) Evaluation of lcr and afd}

From the observation of the curve corresponding to the lcr formula, it is evident that that at both the high and low levels there are few crossings, while the maximum value occurs at 3 $\mathrm{dB}$ below the rms level. Normalization of lcr values is done and represented in terms of crossings per wavelength. Very good agreement between simulated results and theoretical values can be observed beside extremely low levels which are lost in the sampling process.

\section{b) Evaluation of BER}

The study of BPSK over Rayleigh Channel and AWGN channel clearly depicts that the amount of energy required for transmission over AWGN channel is lesser than the energy required for transmission over Rayleigh channel. The study 
also demonstrates that the BER for transmission over AWGN channel is lesser than that for Rayleigh channel. QPSK technique is preferred as compared to BPSK because it has a better BER as compared to BPSK.

\section{CONCLUSION}

This paper elaborates the impact of density variation with regards to the key second order statistics pertaining to flat fading.

In deriving the results based on simulation analysis, various first order and second order statistics have been studied. The focus has been to analyse the second order statistics including lcr and afd and their variations by changing various parameters. Both the theoretical and simulated results have been plotted and goodness of fit is considered. Variations of lcr and afd are calculated in two scenarios and the percentage change has been calculated. It has been seen that by varying the number of scatterers there is a drastic change in the values of second order statistics. BER rate for BPSK channel is evaluated and values of SNR and BER are plotted.The future scope lies in the fact that the first order statistics are insufficient in the analysis the second order statistics can go a long way in improving fading in the channels.Major drawback of complexitity needs to be overcome.

\section{REFERENCES}

[1] Victor M. Hinostroza and Alejandra Mendoza 2007, Second Order Fading Statistics on WWSUS Channels.

[2] .S.Wang,june 2005 On the second order statistics of the instantaneous mutual information of time varying fading channels,ieee,june 2005
[3] .Chang xiang wang, ieee 2007 Level-Crossing Rate and Average Duration of Fades of Deterministic Simulation

[4] Gulzaib rafiq springer,2007, On the first order and second order statistics of the capacity of $n$ nakagami $m$ channels for applications in cooperative networks,

[5] Aleksandra.M.Mitic 2007 Second order statistics of the signal in ricean lognormal fading channel with selection combining.

[6] .Nasiba mandal 2013 vol.1,issue7 Study of higher order statistics in Rayleigh fading channels,

[7] .Zoran hadzi 2006 ieee, Level crossing rate and average fade duration of EGC systems with cochannel interference in Rayleigh fading,

[8] Nikos.C.Sagias 2004 ieee, channel capacity and second order statistics in weibull fading .

[9] Yilmaz,2010 ieee, A new simple model for composite fading channels: Second order statistics and channel capacity

[10] Anjana jain,2011 Estimation of Fading Statistics of Nakagami Channel with Weibull Distributed Tolerable Outage Time.

[11] Yawgeng A. Chau ,2012 On the second order statistics of correlated cascaded Rayleigh fading channels.

[12] Milos Banjdur,2012 Second order statistics of SC Receiver over k- $\mu$ Multipath Fading Channel.

[13] Tasos 2014, A genaralized approach for LCR AFD for. $\alpha-\kappa-\mu$ fading channel. 